\title{
Clinicopathological features and prognosis of papillary thyroid cancer patients with type 2 diabetes mellitus
}

\author{
Ping Shi, Lan Zhang, Yan Liu, Fei Yang, Kai Fu, Ruicong Li, Huijing Shi, Yanzhao Wu \\ Department of Otolaryngology Head and Neck Surgery, The Fourth Hospital of Hebei Medical University and Hebei Tumor Hospital, \\ Shijiazhuang, China \\ Contributions: (I) Conception and design: P Shi, Y Wu, H Shi; (II) Administrative support: Y Wu, H Shi; (III) Provision of study materials or patients: \\ Y Wu, H Shi; (IV) Collection and assembly of data: P Shi, R Li, K Fu; (V) Data analysis and interpretation: P Shi, Y Liu, L Zhang, F Yang; (VI) \\ Manuscript writing: All authors; (VII) Final approval of manuscript: All authors. \\ Correspondence to: Ping Shi, Huijing Shi, Yanzhao Wu. Department of Otolaryngology Head and Neck Surgery, The Fourth Hospital of Hebei \\ Medical University and Hebei Tumor Hospital, Shijiazhuang, China. Email: shirping@hebmu.edu.cn; shj0615@yeah.net; wuyz1116@163.com.
}

\begin{abstract}
Background The clinicopathological characteristics of patients with papillary thyroid carcinoma (PTC) combined with type 2 diabetes mellitus (T2DM) and patients with PTC alone were explored and compared in order to provide a more reasonable treatment plan for the clinical diagnosis and treatment of these patients.

Methods: A total of 1,771 patients from January 2017 to December 2019 who underwent PTC surgery for the first time in the west branch of the Fourth Hospital of Hebei Medical University were analyzed retrospectively. Among them, 111 patients had PTC with T2DM (D-T group) and 1,660 patients had PTC alone (PTC group). The differences in general data and clinical characteristics between the D-T group and the PTC group were analyzed.

Results: There were statistically significant differences in age, gender, and body mass index (BMI) between the D-T group and PTC group $(\mathrm{P}<0.05)$. Multifocality and extra-glandular invasion in the D-T group were significantly higher than the PTC group $(\mathrm{P}<0.05)$. In women, the $\mathrm{D}-\mathrm{T}$ group was older, had a higher $\mathrm{BMI}$, and was more likely to have extra-glandular invasion $(\mathrm{P}<0.05)$ than the PTC group. The prognosis of the D-T group was worse than that of the PTC group as determined by 3 well-developed prognostic staging systems, 8th AJCC TNM staging, MAGIS SCORE, AMES staging, and the results were statistically significant $(\mathrm{P}=0.001, \mathrm{P}<0.001, \mathrm{P}=0.021)$.
\end{abstract}

Conclusions: Our study showed that the overall prognosis of PTC patients with T2DM was worse than that of patients with PTC alone. At the same time, especially in patients younger than 55 years of age, multifocal carcinoma and extra-glandular invasion are likely to occur. In addition, female patients with T2DM are more likely to have extra-glandular invasion than male patients. Therefore, for such patients, preoperative evaluation should be fully conducted.

Koywords: Papillary thyroid carcinoma (PTC); type 2 diabetes; body mass index (BMI); prognosis

Submitted Dec 07, 2021. Accepted for publication Jan 30, 2022.

doi: $10.21037 /$ gs-21-905

View this article at: https://dx.doi.org/10.21037/gs-21-905

\section{Introduction}

Thyroid cancer (TC) accounts for about $1 \%$ of all malignant tumors in the body and $1 / 3$ of head and neck tumors (1), and its incidence is increasing year by year (2). Papillary thyroid carcinoma (PTC) accounts for $80-85 \%$ of TCs (2). Compared with other pathological types of TC, PTC has a lower degree of malignancy, but a higher rate of distant metastasis and recurrence. Furthermore, not all types of PTC have a long survival (3). At present, some studies have found that PTC metastasis, recurrence, and 
prognosis are affected not only by thyroid-related causes, such as extra-glandular invasion of the primary TC and multifocal and lymphatic vascular invasion, but also by its concomitant diseases, which may have a certain impact on the occurrence and progression of TC $(1,3,4)$.

The incidence of type 2 diabetes mellitus (T2DM) has been on the rise in recent years. Studies have confirmed that T2DM is significantly positively correlated with the occurrence and development of liver cancer, kidney cancer, colorectal cancer, and pancreatic cancer, among others $(5,6)$. T2DM and TC are both diseases of the endocrine system. It has been found that the incidence of TC in T2DM is higher than the common incidence $(7,8)$. Study showed that for PTC patients 44 years of age or younger, the increase in the prevalence of diabetes was more than twice expected (9). Currently, it is believed that T2DM's influence on malignant tumors is multi-dimensional, and the related causes of malignant tumors include oxidative stress injury, aggregation of various inflammatory factors, increase of tumor necrosis factor $\alpha$, hyperglycemia and insulin resistance, among others $(6,10)$. Hyperglycemia may be the primary risk factor. A study by the Metabolic Syndrome and Cancer Project, which pooled six prospective cohort, showed that overall cancer rates were positively correlated with blood glucose concentration, but the risk of TC was increased significantly only in men (11). Another study showed that the individuals with high fasting serum glucose levels and abnormal thyroid-stimulating hormone concentration had an increased PTC risk (12). However, there are still few studies on the differences in clinicopathological features between T2DM patients with TC and TC alone, and the conclusions of them are not consistent. Our study is unique in that it is the study to focus exclusively on patients with PTC. The purpose of this study is to investigate the relationship between T2DM patients with PTC and those with PTC alone, especially the difference between sex, age, and prognosis, in order to improve the clinical understanding of the correlation between PTC and T2DM. Meanwhile, in our study, three conventional prognosis systems [AMES (13), MACIS (14) and TNM (15)] were used to estimate the long-term prognosis. This will help clinicians find a more appropriate surgical range, develop reasonable individual surgical plans for patients, and improve their prognosis. We present the following article in accordance with the STROBE reporting checklist (available at https://gs.amegroups.com/article/ view/10.21037/gs-21-905/rc).

\section{Methods}

\section{Patients}

Data from a total of 1,771 hospitalized patients undergoing PTC surgery for the first time from January 2017 to December 2019 in the East District of the Fourth Hospital of Hebei Medical University were collected. Among them, 111 patients were PTC patients with T2DM (D-T group), and the age range was $28-74(\bar{x} \pm \mathrm{SD}, 53.59 \pm 10.25)$ years. At the same time, there were 1,660 patients with PTC alone (PTC group), with an age range of $14-78(\bar{x} \pm$ SD, $44.41 \pm 11.81)$ years.

\section{Clinicopathological characteristics}

The following clinical characteristics of PTC patients were recorded: gender, age at diagnosis, height, weight, body mass index (BMI), and family history. The pathological features of PTC were as follows: maximum diameter of the tumor, multifocal lesions, bilateral lesions, capsular invasion only, extrathyroid extension (ETE), recurrent laryngeal nerve invasion, tracheal invasion, esophageal invasion, combined with Hashimoto's thyroiditis (HT), pathological T stage, and lymph node metastasis. Prognosis was assessed using the American Joint Committee on Cancer (AJCC) $8^{\text {th }}$ edition TNM staging system, the Metastases, Age, Completeness of resection, Invasion, Size (MACIS) scoring system, and the Age, Metastasis, Extent, Size (AMES) clinical staging system. The AMES staging system contains two groups $(13,16)$ : low risk group and high-risk group. Low risk group: (I) for males $<41$ years and females $<51$ years patients, both of them without distant metastasis, and (II) for elderly patients with tumors $<5 \mathrm{~cm}$ with no ETE in PTC. High risk group: (I) younger patients with distant metastasis and (II) for males $\geq 41$ years or females $\geq 51$ years patients with tumors $\geq 5 \mathrm{~cm}$ or ETE in PTC. The MACIS staging system (14) has showed that patients with high score usually would have a poor prognosis. The score is 3.1 (if the patient is $\leq 39$ years) or $0.08 \times$ age (if the patient is $>40$ years) + tumour size $(\mathrm{cm}) \times 0.3+1$ (if the tumour is not completely resectable) +1 (if it is locally invasive) +3 (if it is in the presence of distant metastasis). Four groups should be divided by score: group 1: score $<6$; group 2: score 6-6.99; group 3: score 7-7.99; and group 4 : score $\geq 8$. The differences in various factors between the $\mathrm{D}-\mathrm{T}$ and PTC groups were analyzed. At the same time, the subgroups were defined and compared by age and gender. 
This study was approved by the Ethics Committee of The Fourth Hospital of Hebei Medical University and Hebei Tumor Hospital (No. 2020KS018). All participants fully understood the experimental protocol and signed informed consent forms. All procedures performed in this study were in accordance with the Declaration of Helsinki (as revised in 2013).

\section{Inclusion and exclusion criteria}

The inclusion criteria were as follows: (I) the postoperative pathology of all patients was PTC; (II) all patients underwent unilateral or total thyroidectomy plus preventive neck dissection (including central region dissection \pm lateral neck dissection); (III) there was no history of other malignant tumors; (IV) no history of thyroid or neck surgery; (V) complete clinical and pathological data. The exclusion criteria were as follows: (I) type 1 diabetes mellitus; (II) severe cardiovascular and cerebrovascular diseases or heart, liver, and lung diseases and renal insufficiency. PTC was confirmed by postoperative pathology. T2DM was diagnosed by meeting the latest research criteria of the American Diabetes Association (ADA) and the World Health Organization (WHO).

\section{Statistical methods}

SPSS 24.0 statistical software was used for data processing. Measurement data conforming to a normal distribution were expressed as $\bar{x} \pm \mathrm{SD}$, and the $t$-test was used for comparisons between groups. Nonparametric tests were used for rank data and continuous data with a nonnormal distribution. Statistical data were expressed by percentage or number of cases, and comparison between groups was performed by the $\chi^{2}$ test and Fisher's exact probability method. Logistic regression analysis was used for multivariate analysis. All statistical tests were two-sided probability tests, and $\mathrm{P}<0.05$ was considered statistically significant.

\section{Results}

\section{Analysis of general and clinicopathological features of the overall data}

From January 2017 to December 2019, a total of 1,771 PTC patients undergoing surgery in the Fourth Hospital of Hebei Medical University were enrolled. Among the included patients, 415 were male $(23.4 \%)$ and 1,356 were female
(76.6\%), with an average age of $44.98 \pm 11.98$ years. Multifocal lesions were detected in 630 cases $(35.6 \%)$, bilateral lesions in 434 cases $(24.5 \%)$, thyroid gland invasion in 411 cases (23.2\%), ETE in 355 cases (20.0\%), and positive lymph node metastasis in 924 cases $(52.2 \%)$ during the first operation. Table 1 describes the details of the enrolled patients.

\section{Comparison of the general situation and clinical features between the 2 groups}

There were statistically significant differences in age and gender between the D-T group and the PTC group $(\mathrm{P}<0.05)$, and males and $\geq 55$ years old accounted for a higher proportion in the $\mathrm{D}-\mathrm{T}$ group. The difference in BMI between the D-T group and PTC group was statistically significant $(\mathrm{P}<0.001)$. The proportion of $\mathrm{pT} 1$ tumor stage in the D-T group was significantly lower than that in the PTC group $\left(\chi^{2}=6.058, \mathrm{P}=0.014\right)$. Meanwhile, in terms of ETE, the D-T group was significantly higher than the PTC group $\left(\chi^{2}=5.701, \mathrm{P}=0.017\right)$. In terms of muscle, esophagus, trachea, and recurrent laryngeal nerve invasion, the proportions of the D-T group were higher than those of the PTC group, though differences were not statistically significant. In addition, there were no significant differences in tumor diameter, HT, multifocal lesions, bilateral lesions, and lymph node metastasis. The multivariate logistic regression analysis found that age $(\mathrm{P}=0.001, \mathrm{OR}=3.582)$, gender $(\mathrm{P}=0.015, \mathrm{OR}=1.691)$, and $\mathrm{BMI}(\mathrm{P}=0.018$, $\mathrm{OR}$ $=1.736$ ) were independent factors, and all were positive factors (Table 2).

\section{Influence of age on clinicopathological features}

The 2 groups of patients were divided into groups according to age and analyzed and compared in terms of gender, BMI, maximum tumor diameter, multifocal lesions, bilateral lesions, capsular invasion only, ETE, and lymph node metastasis. The results showed that in the younger age group ( $<55$ years old), the proportion of males in the D-T group was higher $\left(\chi^{2}=8.033, \mathrm{P}=0.005\right)$, and the incidence of multifocal carcinoma in the D-T group was higher $\left(\chi^{2}=4.816, \mathrm{P}=0.028\right)$ than that in the PTC group $\left(\chi^{2}=4.711\right.$, $\mathrm{P}=0.030)$. However, there were no significant differences among the elderly group (Table 3).

\section{Effects of gender on clinicopathological features}

The 2 groups were divided into groups according to sex and 
Table 1 Patients' demographic features and clinicopathological characteristics

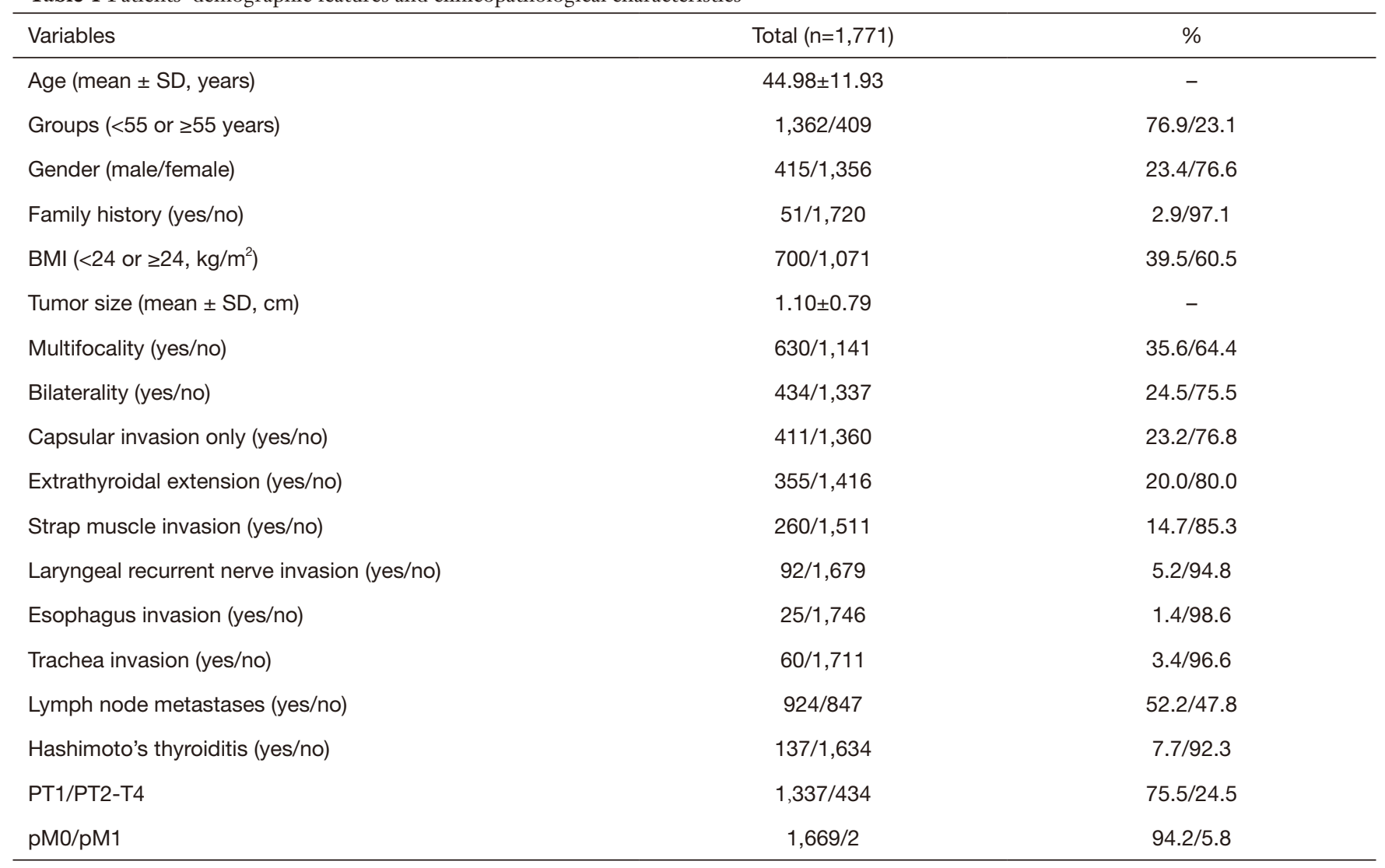

$\mathrm{SD}$, standard deviation; BMI, body mass index.

compared in terms of age, BMI, maximum tumor diameter, multifocal lesions, bilateral lesions, ETE, and lymph node metastasis. The results showed that the D-T group had a higher age $\left(\chi^{2}=43.621, \mathrm{P}<0.001\right)$ and a higher $\mathrm{BMI}\left(\chi^{2}=7.919\right.$, $\mathrm{P}=0.005)$ than the PTC group, with significant differences. At the same time, ETE was mainly found in women. Among women, the incidence of ETE was higher in the D-T group than that in the PTC group, and the difference was statistically significant $\left(\chi^{2}=6.633, \mathrm{P}=0.010\right)$. In males, there was only a difference in age between the 2 groups. There were also differences in BMI and ETE percentages, both of which were higher than the PTC group, but these did not reach significance (Table 4).

\section{Evaluation of expected outcomes of the 2 groups using different staging or scoring systems}

Prognosis was assessed using 3 well-developed prognostic staging systems. The 2 groups were statistically significant in terms of the 3 staging systems, $8^{\text {th }}$ AJCC TNM staging,
MAGIS SCORE, AMES staging $(\mathrm{P}=0.001, \mathrm{P}<0.001$, and $\mathrm{P}=0.021$ ) (Table 5). However, when prognosis was assessed by different sex, there was a big difference between female and male groups in terms of the MAGIS SCORE and AMES staging. The female group was statistically significant in terms of those two staging systems $\left(\chi^{2}=5.286\right.$, $\mathrm{P}=0.021$ and $\left.\chi^{2}=15.236, \mathrm{P}<0.001\right)$, but the male group was not $\left(\chi^{2}=3.744, \mathrm{P}=0.053\right.$ and $\left.\chi^{2}=0.346, \mathrm{P}=0.557\right)$. However, when patients were assessed using the $8^{\text {th }}$ edition TNM staging system, whatever in all patients, the female group or the male group, the differences were statistically significant $(\mathrm{P}<0.001, \mathrm{P}=0.001$ and $\mathrm{P}<0.001 ;$ Tables 6,7).

\section{Discussion}

T2DM is a common endocrine disease, and studies in recent years have suggested that $\mathrm{T} 2 \mathrm{DM}$ is significantly correlated with the occurrence of TC $(6,17)$ According to current studies $(6,10)$, abnormal glucose metabolism caused by T2DM may be the main reason for its influence on 
Table 2 Clinicopathological characteristics of 1,771 PTC patients with and without T2DM

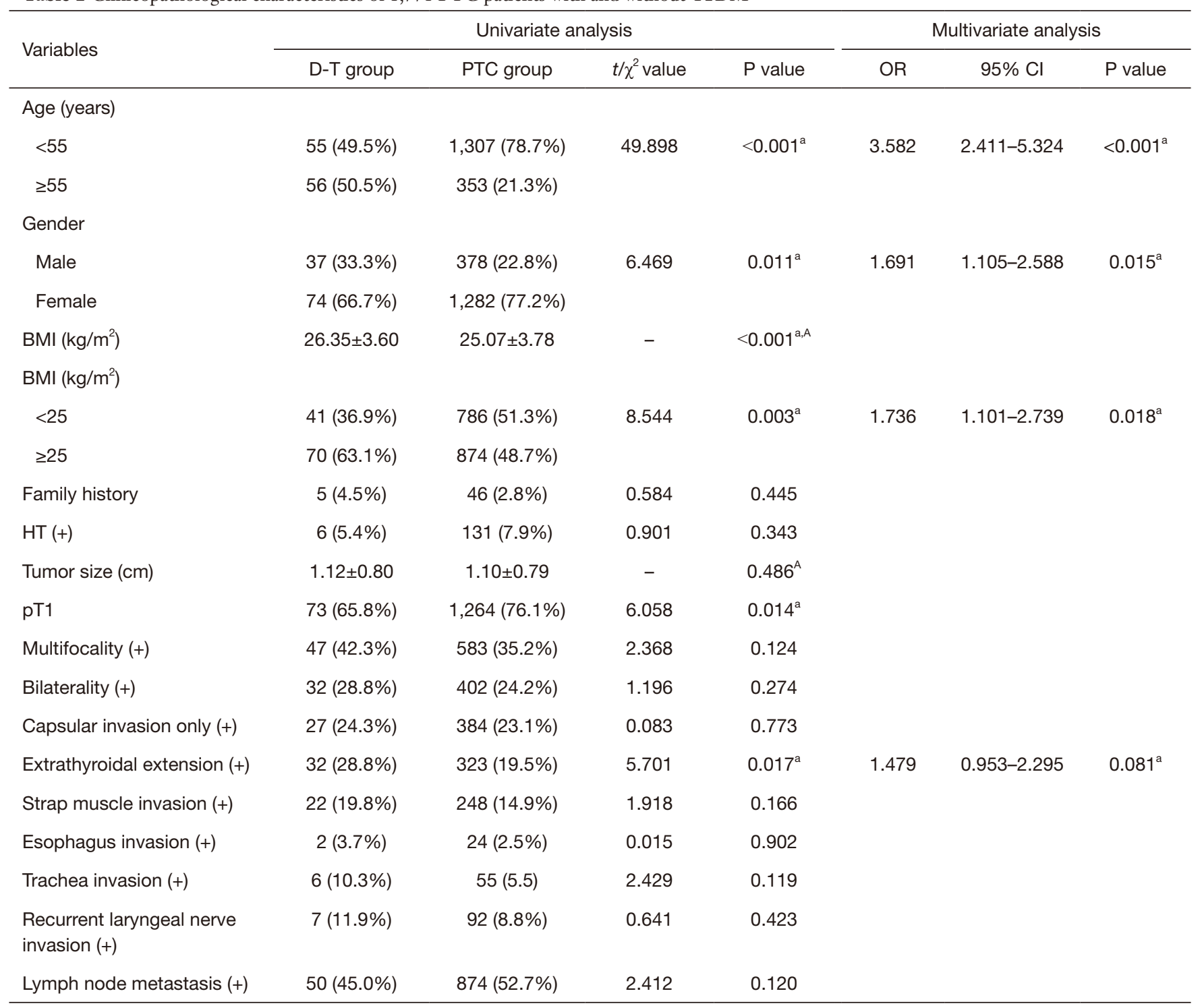

${ }^{\text {a }}$, statistically significant $(\mathrm{P}<0.05)$. All calculated using two-tailed Student's $t$-test. ${ }^{A}$, calculated using the $t$-test. Others calculated using the chi-square test. OR, odds ratio; Cl, confidence interval; ETE, extrathyroidal extension; BMI, body mass index; HT, Hashimoto's thyroiditis; T2DM, type 2 diabetes mellitus; PTC, papillary thyroid carcinoma.

the occurrence and development of TC. In this study, the differences in age and proportion of stage I disease between patients with PTC combined with T2DM and patients with PTC alone were similar to those in previous studies (18). In the AJCC TNM staging system, the age at which a patient is initially diagnosed with PTC is an important prognostic parameter (19). In the recently updated $8^{\text {th }}$ edition, the age of PTC staging was raised from 45 to 55 years, suggesting that the age of PTC patients should be paid attention to. At the same time, previous studies have shown that in PTC, males are more prone to bilateral and multifocal carcinoma and cervical lymph node metastasis than females $(1,17,20)$. In this study, it was found that among patients younger than 55 years old, there were more male patients with PTC complicated with diabetes than patients with PTC alone, and they were more prone to multiple foci and extraglandular invasion. Therefore, sufficient attention should be paid to male patients with PTC complicated with diabetes, especially those under 55 years old.

In addition, in terms of gender, we found significant 
Table 3 The influence of age on pathological characteristics

\begin{tabular}{|c|c|c|c|c|c|c|c|c|}
\hline Variables & \multicolumn{3}{|c|}{ D-T 111} & \multicolumn{5}{|c|}{ PTC 1660} \\
\hline Gender & & & 0.140 & & & 0.291 & $0.005^{\mathrm{a}}$ & 0.302 \\
\hline Male & $22(40 \%)$ & $15(26.8 \%)$ & & $305(23.3 \%)$ & $73(20.7 \%)$ & & & \\
\hline Female & $33(60 \%)$ & $41(73.2 \%)$ & & $1,002(76.7 \%)$ & $280(79.3 \%)$ & & & \\
\hline Multifocality (+) & $27(49.1 \%)$ & $20(35.7 \%)$ & 0.154 & $453(34.7 \%)$ & $130(36.8 \%)$ & 0.449 & 0.028 & 0.872 \\
\hline Capsular invasion only (+) & $12(21.8 \%)$ & $15(26.8 \%)$ & 0.542 & 307 (23.5\%) & $77(21.8 \%)$ & 0.508 & 0.774 & 0.408 \\
\hline Extrathyroidal extension (+) & $16(29.1 \%)$ & $16(28.6 \%)$ & 0.952 & $230(17.6 \%)$ & $93(26.3 \%)$ & $<0.001^{\mathrm{a}}$ & $0.030^{\mathrm{a}}$ & 0.416 \\
\hline Bilaterality (+) & $18(32.7 \%)$ & $14(25 \%)$ & 0.369 & $302(23.1 \%)$ & $100(28.3 \%)$ & $0.042^{\mathrm{a}}$ & 0.099 & 0.606 \\
\hline
\end{tabular}

Data are $\mathrm{n}(\%)$ or mean \pm SD. P1 = A group vs. B group; P2 = C group vs. D group; P3 = A group vs. C group; P4 = B group vs. D group. ${ }^{a}$, statistically significant $(\mathrm{P}<0.05)$. All calculated using two-tailed Student's $t$-test. All calculated using the chi-square test. D-T: D-T group (PTC patients with T2DM). PTC: PTC group (patients with PTC alone).

Table 4 The influence of sex on pathological characteristics

\begin{tabular}{|c|c|c|c|c|c|c|c|c|}
\hline Variables & \multicolumn{3}{|c|}{ D-T 111} & \multicolumn{5}{|c|}{ PTC 1660} \\
\hline \multicolumn{9}{|l|}{ Age, years } \\
\hline$<55$ & $22(59.5)$ & $33(44.6)$ & 0.140 & $305(80.7)$ & $1,002(78.2)$ & 0.291 & $0.003^{\mathrm{a}}$ & $<0.001^{\mathrm{a}}$ \\
\hline$\geq 55$ & $15(40.5)$ & $41(55.4)$ & & $73(19.3)$ & $280(22.8)$ & & & \\
\hline$<24$ & $6(16.2)$ & $21(28.4)$ & 0.159 & $95(25.1)$ & $578(45.1)$ & $<0.001^{\mathrm{a}}$ & 0.228 & $0.005^{\mathrm{a}}$ \\
\hline$\geq 24$ & $31(83.8)$ & $53(71.6)$ & & $283(74.9)$ & $704(54.9)$ & & & \\
\hline \multicolumn{9}{|l|}{ Diameter (cm) } \\
\hline$\leq 1$ & $20(54.1)$ & $51(68.9)$ & 0.124 & $241(63.8)$ & $850(66.3)$ & 0.359 & 0.244 & 0.643 \\
\hline Bilaterality (+) & $13(35.1)$ & $19(25.7)$ & 0.300 & $96(25.4)$ & $306(23.9)$ & 0.542 & 0.199 & 0.732 \\
\hline Capsular invasion only (+) & $10(27.0)$ & $17(22.1)$ & 0.561 & $90(23.8)$ & $294(22.9)$ & 0.722 & 0.662 & 0.862 \\
\hline Extrathyroidal extension (+) & $8(21.6)$ & $24(32.4)$ & 0.236 & $67(17.8)$ & $256(20.0)$ & 0.333 & 0.557 & $0.010^{\mathrm{a}}$ \\
\hline Lymph node metastasis (+) & $23(62.2)$ & $27(36.5)$ & 0.010 & $263(69.6)$ & $611(47.7)$ & $<0.001^{\mathrm{a}}$ & 0.352 & 0.061 \\
\hline
\end{tabular}

P1 = A group vs. B group; P2 = C group vs. D group; P3 = A group vs. C group; P4 = B group vs. D group. ${ }^{a}$, statistically significant (P<0.05). All calculated using two-tailed Student's $t$-test. All calculated using the chi-square test. D-T, D-T group (PTC patients with T2DM); PTC, PTC group (patients with PTC alone); BMI, body mass index. 
Table 5 Evaluation of the expected prognostic outcomes of the 2 groups using different staging or scoring systems

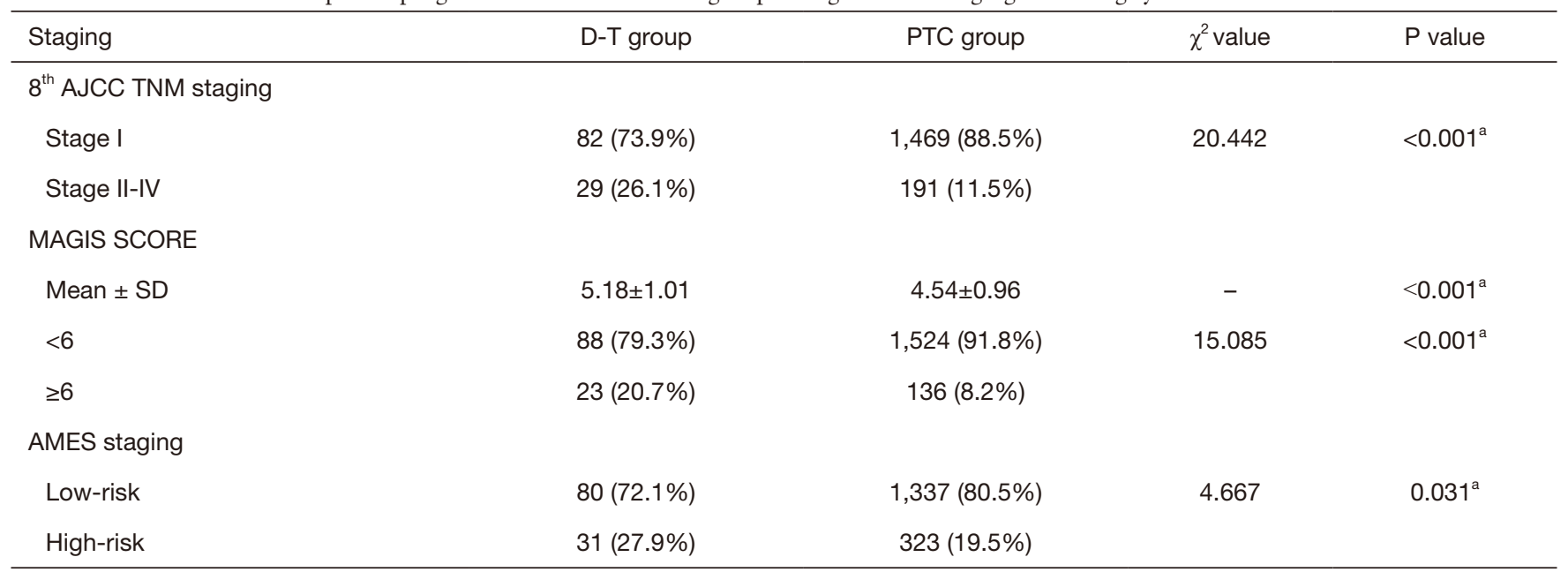

Data are $\mathrm{n}(\%)$ or mean $\pm \mathrm{SD}$. ${ }^{a}$, statistically significant $(\mathrm{P}<0.05)$. All calculated using two-tailed Student's $t$-test. All calculated using the chi-square test. D-T group, PTC patients with T2DM. PTC group, patients with PTC alone. AJCC, American Joint Committee on Cancer.

Table 6 Evaluation of the expected prognostic outcomes of the 2 groups by different sex using different staging or scoring systems (female)

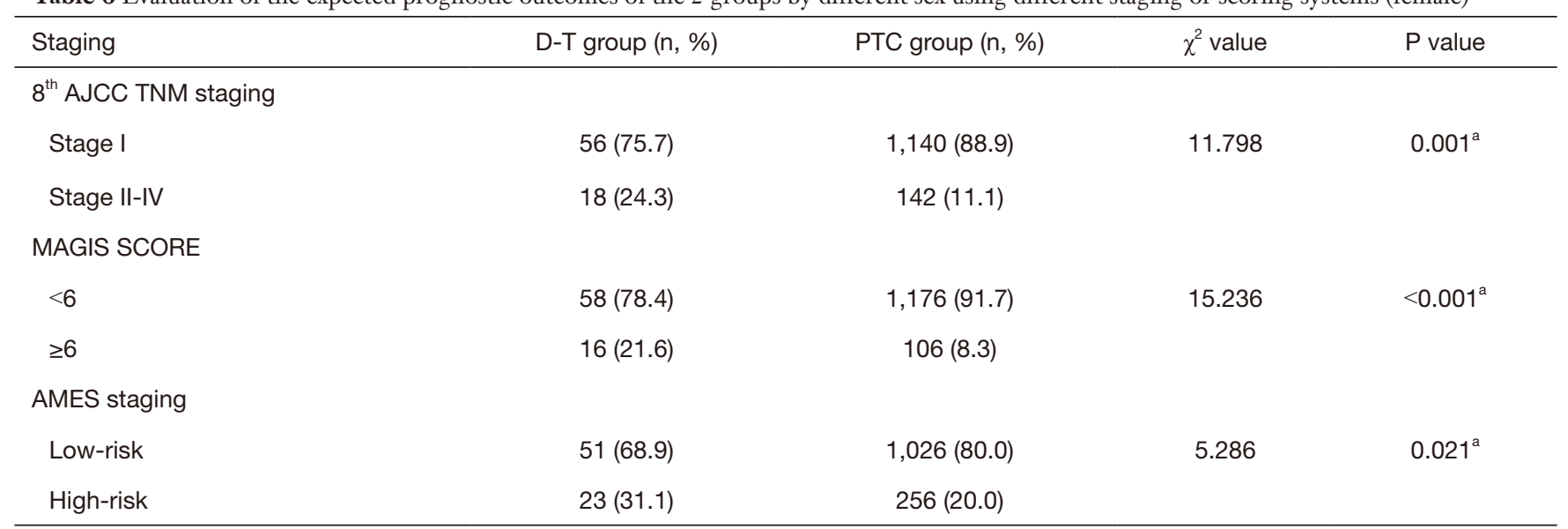

a , statistically significant $(\mathrm{P}<0.05)$. All calculated using two-tailed Student's $t$-test. All calculated using the chi-square test. $\mathrm{D}-\mathrm{T}$ group, $\mathrm{PTC}$ patients with T2DM; PTC group, patients with PTC alone; AJCC, American Joint Committee on Cancer.

differences between the D-T group and the PTC group. The D-T group had a higher proportion of males, which was similar to the research results of Deng et al. (21). Gender plays an important role in TC. As early as more than 10 years ago, Machens et al. (22) showed that the prognosis of male TC was worse, which was considered to be related to distant metastasis. Recently, it has been found that male PTC is a more aggressive disease, as male patients have a larger tumor diameter and are more prone to cervical lymph node metastasis than females, with a higher incidence of advanced lesions and prognosis (20). Lee et al. (17) showed that male was also an independent risk factor for postoperative recurrence in patients with $\mathrm{PTC}>1 \mathrm{~cm}$. Therefore, we believe that gender plays an important role in TC. By further grouping and comparing the 2 groups according to different genders, the results showed that the effect of T2DM on PTC patients was mainly concentrated in women, the elderly, and overweight patients. In women, the proportion of patients with T2DM was higher. The difference was only significant in men by age.

In this study, compared with the PTC group, ETE showed significant differences between the 2 groups, and extra-glandular extension has always been considered as a factor affecting the prognosis of well-differentiated TC (23). 
Table 7 Evaluation of the expected prognostic outcomes of the 2 groups by different sex using different staging or scoring systems (male)

\begin{tabular}{lccc}
\hline Staging & D-T group $(\mathrm{n}, \%)$ & PTC group $(\mathrm{n}, \%)$ & $\chi^{2}$ value \\
\hline $8^{\text {th }}$ AJCC TNM staging & $23(62.2)$ & $329(87.0)$ & 16.195 \\
Stage I & $14(37.8)$ & $49(13.0)$ & $3.001^{\text {a }}$ \\
Stage II-IV & & & $3444(92.1)$ \\
MAGIS score & $30(81.1)$ & $30(7.9)$ & 0.053 \\
$<6$ & $7(18.9)$ & & 0.346 \\
$\geq 6$ & & $311(82.3)$ & 0.557 \\
AMES staging & $29(78.4)$ & $67(17.7)$ & \\
Low-risk & $8(21.6)$ & & \\
High-risk & & & \\
\hline
\end{tabular}

${ }^{a}$, statistically significant $(\mathrm{P}<0.05)$. All calculated using two-tailed Student's $t$-test. All calculated using the chi-square test. D-T group, PTC patients with T2DM; PTC group, patients with PTC alone; AJCC, American Joint Committee on Cancer.

In this study, combined with the $8^{\text {th }}$ edition of the AJCC TNM staging system, we divided extra-glandular invasion into 2 categories: simple invasion (capsule invasion) and extra-glandular extension (including muscle, trachea, esophagus, and recurrent laryngeal nerve invasion). This new classification method effectively improved the inconsistency between pathologists and observers in reporting microscopic ETE. The surgeon's intraoperative ETE report was included in the final tumor staging. Previous research (23) has shown that extra-glandular extension is of great significance for the prognosis of TC, which is more prone to invasion, metastasis, recurrence, and worse prognosis. In this study, we found that the presence of T2DM significantly increased the degree of invasion in female patients, especially for the invasion of extraglandular muscle and invasion beyond the strap muscles, and the proportion of the patients with extrathyroidal extension also increased significantly. Overall, although the proportion of T2DM patients with PTC is high in males, its influence on females is greater, and the proportion of patients with advanced age and BMI is higher. At the same time, extra-glandular extension is more likely to occur, which may lead to more distant metastasis and recurrence, suggesting the need for a more aggressive approach to clinical surgical procedures.

A study has shown that BMI is associated with the risk of TC, and the higher the BMI, the greater the risk (24). In men, obesity is associated with an increased risk of TC, and obesity itself is an independent risk factor for TC. At the same time, the risk of diabetic patients suffering from various types of cancer has been increasing, followed by an increased risk of cancer death (25). Oberman et al. showed that obesity and diabetes were significantly correlated with the occurrence of differentiated thyroid cancer (DTC) to a certain extent. In particular, BMI was a strong predictor of DTC (26). Therefore, despite potential mechanisms such as insulin resistance and sex hormone production disorders, obesity may also be behind the association between diabetes and cancer. In this study, we found significant differences in BMI between the D-T group and the PTC group. Chen et al. showed that the percentage of disease progression in TC patients with T2DM was higher than that in nondiabetic patients (18). At the same time, the overexpression of estrogen receptors in TC cells may be one of the reasons for the high incidence of TC in women. Estrogen can enhance the transcription of target genes, thus leading to the proliferation and differentiation of TC cells (27). In this study, the proportion of overweight patients out of female patients with T2DM was significantly higher than that in the PTC group. Combined with previous studies, we believe that this is also one of the reasons for the increase of ETE in female T2DM patients. Combined with the results of this study, obesity in diabetic patients not only increases the incidence of PTC, but also promotes the progression of PTC, enhances its invasiveness, and worsens the prognosis. The reason may be related to the imbalance of sex hormones caused by diabetes. Therefore, in clinical practice, more attention should be paid to the relationship between tumor location and surrounding structures in overweight female patients with PTC complicated with T2DM. In view 
of its invasiveness, for patients with invasion at dangerous locations suggested by ultrasound, such as near the trachea, nerves, and the esophagus, puncture examination and surgical treatment should be performed at an early stage.

In view of the short follow-up time of this study, we used 3 scoring systems to analyze and evaluate prognosis. Currently, AMES staging and the MACIS scoring system are the most commonly used methods to predict survival and develop selective treatment strategies for patients with TC. Previous study has shown that T2DM leads to high cancer-related mortality in patients with DTC (25). At the same time, the total mortality of TC patients with T2DM has increased (18). The expected outcomes of this study were consistent with these results. At the same time, we conducted a gender-specific re-evaluation using AMES and MACIS scores which showed that the prognosis of female patients with diabetic PTC was worse than that of female patients with PTC alone, while there was no significant difference in male patients. Recent study has shown that obesity and T2DM do not affect the prognosis of patients with DTC (28). Combined with this study, we concluded that the impact of T2DM on the prognosis of male patients was not significant, but for the prognosis of female patients, the expected score was still very different. Typically, thyroxine supplementation is required after surgery and the recurrence rate is reduced primarily through long-term TSH hormone suppression therapy (15). Combined with the results of this study, patients with T2DM, especially female patients, have a poor prognosis. Therefore, in addition to TSH hormone suppression therapy, attention should be paid to the monitoring of blood glucose. At the same time, according to the guidelines of American Diabetes Association (ADA) and Chinese Diabetes Society (CDS), the combination of diet, exercise, and drugs, to give up smoking and alcohol, control weight could be used to control the blood glucose in the PTC patients with T2DM.

The male sample size in this study was small, and this was a retrospective study. Meanwhile, due to the short follow-up period, no real prognostic analysis was conducted, which may have resulted in biased results that cannot fully reveal the real situation. In the future, the sample size will continue to be expanded, and a prospective study will be conducted in combination with clinical practice to verify the conclusions.

\section{Conclusions}

Our study showed that among PTC patients with T2DM, patients $<55$ years of age were more likely to have multifocal lesions and extra-glandular extension. In women, the incidence of extra-glandular extension was significantly increased and the prognosis was worse. Therefore, for such patients, preoperative evaluation should be fully conducted, early operation should be performed, careful operation of the surgical margin should be paid attention to during the operation, and total resection plus preventive central region dissection should be performed when necessary, in order to minimize the recurrence rate and improve the prognosis of such patients.

\section{Acknowledgments}

The authors thank the patients and healthy volunteers for their cooperation and participation in the study.

Funding: This study was supported by the Hebei Province Natural Scientific Fund (No. H2020206273) and Department of Health of Hebei Province Grant (No. 20190085).

\section{Footnote}

Reporting Checklist: The authors have completed the STROBE reporting checklist. Available at https:// gs.amegroups.com/article/view/10.21037/gs-21-905/rc

Data Sharing Statement: Available at https://gs.amegroups. com/article/view/10.21037/gs-21-905/dss

Conflicts of Interest: All authors have completed the ICMJE uniform disclosure form (available at https://gs.amegroups. com/article/view/10.21037/gs-21-905/coif). The authors have no conflicts of interest to declare.

Ethical Statement: The authors are accountable for all aspects of the work in ensuring that questions related to the accuracy or integrity of any part of the work are appropriately investigated and resolved. This study was approved by the Ethics Committee of The Fourth Hospital of Hebei Medical University and Hebei Tumor Hospital (No. 2020KS018). All participants fully understood the experimental protocol and signed informed consent forms. All procedures performed in this study were in accordance with the Declaration of Helsinki (as revised in 2013).

Open Access Statement: This is an Open Access article distributed in accordance with the Creative Commons 
Attribution-NonCommercial-NoDerivs 4.0 International License (CC BY-NC-ND 4.0), which permits the noncommercial replication and distribution of the article with the strict proviso that no changes or edits are made and the original work is properly cited (including links to both the formal publication through the relevant DOI and the license). See: https://creativecommons.org/licenses/by-nc-nd/4.0/.

\section{References}

1. Ahn BH, Kim JR, Jeong HC, et al. Predictive factors of central lymph node metastasis in papillary thyroid carcinoma. Ann Surg Treat Res 2015;88:63-8.

2. Siegel RL, Miller KD, Jemal A. Cancer statistics, 2018. CA Cancer J Clin 2018;68:7-30.

3. Ho AS, Luu M, Barrios L, et al. Incidence and Mortality Risk Spectrum Across Aggressive Variants of Papillary Thyroid Carcinoma. JAMA Oncol 2020;6:706-13.

4. Kuo JH, Chabot JA, Lee JA. Breast cancer in thyroid cancer survivors: An analysis of the Surveillance, Epidemiology, and End Results-9 database. Surgery 2016;159:23-9.

5. Hu Y, Zhang X, Ma Y, et al. Incident Type 2 Diabetes Duration and Cancer Risk: A Prospective Study in Two US Cohorts. J Natl Cancer Inst 2021;113:381-9.

6. Fransgaard T, Hallas J, Thygesen LC, et al. Association between diabetes and oncological outcomes in patients undergoing neoadjuvant chemo-radiotherapy for rectal cancer. Surg Oncol 2019;28:62-6.

7. Zivaljevic V, Vlajinac H, Jankovic R, et al. Case-control study of anaplastic thyroid cancer. Tumori 2004;90:9-12.

8. Aschebrook-Kilfoy B, Sabra MM, Brenner A, et al. Diabetes and thyroid cancer risk in the National Institutes of Health-AARP Diet and Health Study. Thyroid 2011;21:957-63.

9. Paulus YM, Riedel ER, Sabra MM, et al. Prevalence of diabetes mellitus in patients with newly evaluated papillary thyroid cancer. Thyroid Res 2014;7:7.

10. Vella V, Malaguarnera R. The Emerging Role of Insulin Receptor Isoforms in Thyroid Cancer: Clinical Implications and New Perspectives. Int J Mol Sci 2018;19:3814.

11. Stocks T, Rapp K, Bjørge T, et al. Blood glucose and risk of incident and fatal cancer in the metabolic syndrome and cancer project (me-can): analysis of six prospective cohorts. PLoS Med 2009;6:e1000201.

12. Hu MJ, Zhang C, Liang L, et al. Fasting serum glucose, thyroid-stimulating hormone, and thyroid hormones and risk of papillary thyroid cancer: A case-control study. Head Neck 2019;41:2277-84.

13. Cady B, Rossi R. An expanded view of risk-group definition in differentiated thyroid carcinoma. Surgery 1988;104:947-53.

14. Hay ID, Bergstralh EJ, Goellner JR, et al. Predicting outcome in papillary thyroid carcinoma: development of a reliable prognostic scoring system in a cohort of 1779 patients surgically treated at one institution during 1940 through 1989. Surgery 1993;114:1050-7; discussion 1057-8.

15. Tuttle RM, Haugen B, Perrier ND. Updated American Joint Committee on Cancer/Tumor-Node-Metastasis Staging System for Differentiated and Anaplastic Thyroid Cancer (Eighth Edition): What Changed and Why? Thyroid 2017;27:751-6.

16. Sanders LE, Cady B. Differentiated thyroid cancer: reexamination of risk groups and outcome of treatment. Arch Surg 1998;133:419-25.

17. Lee YH, Lee YM, Sung TY, et al. Is Male Gender a Prognostic Factor for Papillary Thyroid Microcarcinoma? Ann Surg Oncol 2017;24:1958-64.

18. Chen ST, Hsueh C, Chiou WK, et al. Disease-specific mortality and secondary primary cancer in welldifferentiated thyroid cancer with type 2 diabetes mellitus. PLoS One 2013;8:e55179.

19. Adam MA, Thomas S, Hyslop T, et al. Exploring the Relationship Between Patient Age and Cancer-Specific Survival in Papillary Thyroid Cancer: Rethinking Current Staging Systems. J Clin Oncol 2016;34:4415-20.

20. Ding J, Wu W, Fang J, et al. Male sex is associated with aggressive behaviour and poor prognosis in Chinese papillary thyroid carcinoma. Sci Rep 2020;10:4141.

21. Deng Y, Ke S, Liu H, et al. Analysis of clinicopathologic features in patients with comorbid thyroid cancer and diabetes mellitus. Chinese Journal of General Surgery 2020;29:565-73.

22. Machens A, Hauptmann S, Dralle H. Disparities between male and female patients with thyroid cancers: sex difference or gender divide? Clin Endocrinol (Oxf) 2006;65:500-5.

23. McConahey WM, Hay ID, Woolner LB, et al. Papillary thyroid cancer treated at the Mayo Clinic, 1946 through 1970: initial manifestations, pathologic findings, therapy, and outcome. Mayo Clin Proc 1986;61:978-96.

24. Kwon H, Chang Y, Cho A, et al. Metabolic Obesity Phenotypes and Thyroid Cancer Risk: A Cohort Study. Thyroid 2019;29:349-58. 
25. Garg SK, Maurer H, Reed K, et al. Diabetes and cancer: two diseases with obesity as a common risk factor. Diabetes Obes Metab 2014;16:97-110.

26. Oberman B, Khaku A, Camacho F, et al. Relationship between obesity, diabetes and the risk of thyroid cancer. Am J Otolaryngol 2015;36:535-41.

27. Zane M, Parello C, Pennelli G, et al. Estrogen and thyroid cancer is a stem affair: A preliminary study. Biomed Pharmacother 2017;85:399-411.

28. Elbasan O, Yavuz DG. Effects of concomitant obesity and diabetes on the aggressiveness and outcomes of differentiated thyroid cancer patients. Arch Endocrinol Metab 2021;65:455-61.
Cite this article as: Shi P, Zhang L, Liu Y, Yang F, Fu K, Li R, Shi H, Wu Y. Clinicopathological features and prognosis of papillary thyroid cancer patients with type 2 diabetes mellitus. Gland Surg 2022;11(2):358-368. doi: 10.21037/gs-21-905 